\title{
Prophylaxis for Venous Thromboembolism Following Total Knee Arthroplasty: A Survey of Korean Knee Surgeons
}

\author{
Nam Ki Kim, MD 1 , Tae Kyun Kim, $\mathrm{MD}^{2}$, Jong Min Kim, $\mathrm{MD}^{3}$, and Churl Hong Chun, $\mathrm{MD}^{4}$ \\ ${ }^{1}$ Department of Orthopaedic Surgery, Medi-Yin Hospital, Paju; ${ }^{2}$ Department of Orthopaedic Surgery, Seoul National University Bundang Hospital, Seongnam; \\ ${ }^{3}$ Department of Orthopaedic Surgery, Asan Medical Center, Seoul; ${ }^{4}$ Department of Orthopaedic Surgery, Wonkwang University School of Medicine, Iksan, Korea
}

Purpose: The purpose of this study is to provide information on the actual status and prevailing trend of prophylaxis for venous thromboembolism (VTE) following total knee arthroplasty (TKA) in South Korea.

Materials and Methods: The Korean Knee Society (KKS) developed a questionnaire with 6 clinical questions on VTE. The questionnaire was distributed to all members of KKS by both postal and online mail. Participants were asked to supply details on their specialty and to select methods of prophylaxis they employ. Of the total members of KKS, 27.9\% participated in the survey.

Results: The percentage of surgeons who routinely performed prophylaxis for VTE was $60.4 \% ; 19.4 \%$ performed prophylaxis depending on the patient's health condition; and the remaining $20.2 \%$ never implemented prophylaxis after surgery. The common prophylactic methods among the responders were compression stocking (72.9\%), pneumatic leg compression (63.3\%), perioral direct factor Xa inhibitor (46.9\%), and low-molecularweight heparin (39.5\%). For the respondents who did not perform prophylaxis, the main reason (51.5\%) was the low risk of postoperative VTE considering the low incidences in Asians.

Conclusions: The present study involving members of the KKS will help to comprehend the actual status of VTE prevention in South Korea. The results of this study may be useful to design VTE guidelines appropriate for Koreans in the future.

Keywords: Knee, Arthroplasty, Thromboembolism, Prophylaxis, Survey

\section{Introduction}

Major orthopedic surgeries, especially arthroplasties of the lower extremities, are high-risk surgeries for venous thromboembolism (VTE) including deep venous thrombosis (DVT) and pulmonary embolism ${ }^{1,2)}$. Without the use of any mechanical or pharmaceutical prophylaxis after lower limb arthroplasty, the overall incidence of DVT after total knee arthroplasty (TKA)

Received January 11, 2016; Revised May 1, 2016; Accepted May 22, 2016 Correspondence to: Churl Hong Chun, MD

Department of Orthopaedic Surgery, Wonkwang University School of Medicine, 895 Muwang-ro, Iksan 54538, Korea

Tel: +82-63-859-1363, Fax: +82-63-852-9329

E-mail: cch@wonkwang.ac.kr

This is an Open Access article distributed under the terms of the Creative Commons Attribution Non-Commercial License (http://creativecommons.org/licenses/by-nc/4.0/) which permits unrestricted non-commercial use, distribution, and reproduction in any medium, provided the original work is properly cited. ranges from $40 \%$ to $84 \%^{3,4)}$. The incidence of DVT reported in South Korea ranges from $15.7 \%$ to $41.8 \%^{5,6}$. Previous clinical trials have shown that asymptomatic DVT after major joint surgery can be significantly reduced with VTE prophylaxis to around $20 \%$ to $40 \%^{7,8)}$

As VTE is a highly prevalent and potentially lethal complication, various prophylactic methods to prevent the development of DVT are currently available. However, the need for prophylaxis to prevent DVT after TKA and the efficacy of each method are disputed, and there is still no consensus.

In other countries, survey-based studies have been conducted on the actual status of prophylaxis performed by surgeons. Additionally, several countries developed guidelines for prophylaxis after arthroplasty to prevent VTE. The actual status of prophylaxis for VTE in South Korea is assumed to differ from that in other countries owing to racial and healthcare system differences. In South Korea, however, prophylaxis implementation and types in practical clinical settings have not yet been clearly investi- 
gated $^{9-11)}$. There are still no prophylaxis guidelines appropriate to the clinical circumstances in South Korea. The Korean Knee Society (KKS) recognized the necessity of investigating the actual status on the use of prophylaxis for VTE in South Korea. Therefore, the objectives of this study are to investigate the proportion of orthopedists who provide DVT prophylaxis among those who conduct TKA in South Korea as well as the prophylactic methods they use and the rationale of physicians who do not provide prophylaxis. To our knowledge, this is the first study to survey the state of prophylaxis implementation across all members of the KSS. Therefore, the results of this study will provide accurate data regarding the rate of prophylaxis after TKA and the methods used in the actual Korean clinical environment. Furthermore, this study is expected to help the establishment of guidelines appropriate to the circumstances in South Korea. The hypothesis of this research is that prophylaxis is implemented by more than $50 \%$ of medical practitioners who perform TKA in Korea in order to prevent VTE, with mechanical prevention being the most preferred method.

\section{Materials and Methods}

The VTE task force team of the KKS designed the questionnaire used in the survey of this study. The questionnaire was written in Korean and composed of 6 questions (Appendix 1). The major elements included in the questionnaire are as follows: implementation of prophylactic treatment for VTE, prophylactic methods, and rationale for not performing prophylaxis as applicable. The prophylactic methods for DVT included in the study were pharmaceutical therapies (aspirin, warfarin, low-molecular-weight heparin [LMWH], perioral factor Xa inhibitor, and intravenous factor Xa inhibitor) and mechanical therapies (compression stocking and intermittent pneumatic pumps). The questionnaire was sent via both email and postal mail to a unique group of orthopedic surgeons who are members of the KKS and perform TKA, and their replies were returned in the same manner. The survey was performed for 3 months from December 2011 to February 2012.

The questionnaires were distributed to all registered members of the KSS who specialize in the knee joint ( $\mathrm{n}=796)$. Of the 796 orthopedic surgeons who received the questionnaire, 222 participated in the survey. The response rate was $27.9 \%$.

The 222 orthopedic surgeons who participated in the survey were composed of professors at university hospitals (40.5\%), fellows at university hospitals (2.7\%), and surgeons in general (19.4\%), private (32\%), and public hospitals (5.4\%) (Fig. 1).
Of the responding surgeons, $9.9 \%$ performed more than 300 surgeries a year. In particular, $27.5 \%$ and $18.5 \%$ of them performed 100-300 TKAs and 50-100 TKAs a year, respectively. Of the respondents, $44.1 \%$ conducted TKAs less than 50 times a year.

Among the responding surgeons, $42 \%$ attended more than 500 cumulative cases of TKA, and $12.1 \%$ and $18.9 \%$ attended $300-$ 500 cases and $100-300$ cases, respectively. Furthermore, 27\% of the respondents attended less than 100 cumulative cases of TKA.

\section{Results}

In this survey, the percentage of orthopedic surgeons who routinely performed prophylaxis for VTE after TKA was $60.4 \%$, and $19.4 \%$ of the surgeons performed prophylaxis depending on the patient's health condition.

Prophylaxis was performed after TKA for patients who did not present with any accompanying diseases. However, prophylaxis was not performed in patients at high risk for hemorrhage due to administration of anticoagulants for treatment of cardiovascular or cerebrovascular disease or in patients suffering from any blood disorder that impedes normal hemostasis. The remaining 20.2\% of the surgeons never implemented prophylaxis after surgery (Fig. 2).

The reasons for no use of prophylaxis among the $20.2 \%$ of the surgeons were as follows: 1) the low incidence of VTE after joint arthroplasty, 2) risk of complications related to the preventive treatment for VTE (bleeding, postoperative infection, delayed wound healing, etc.), 3) ignorance of the procedure despite the awareness of the necessity of VTE prophylaxis after joint arthroplasty, and 4) others. The primary reason for not performing prophylaxis reported by $51.1 \%$ of the surgeons in this group was the low incidence of VTE. The risk of complication by prophylactic

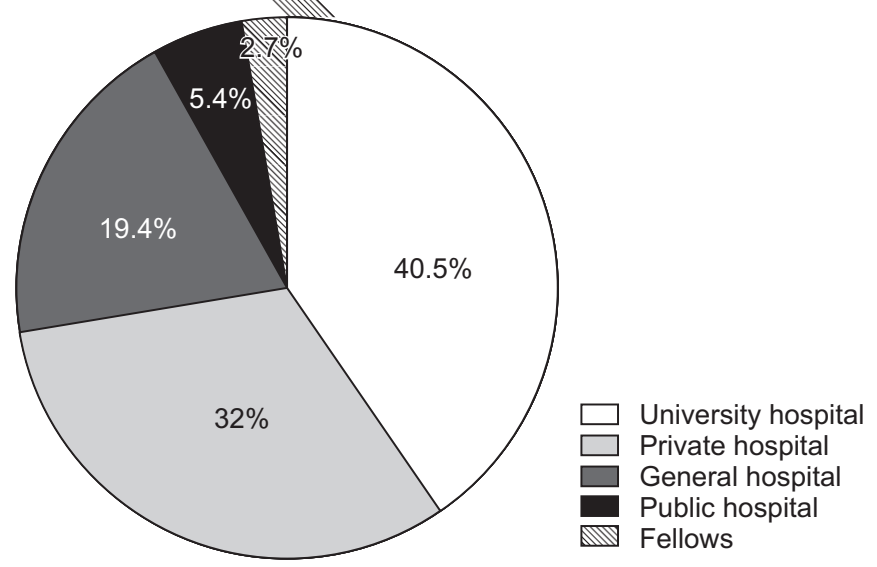

Fig. 1. Pie diagram showing the composition of respondents of the survey. 


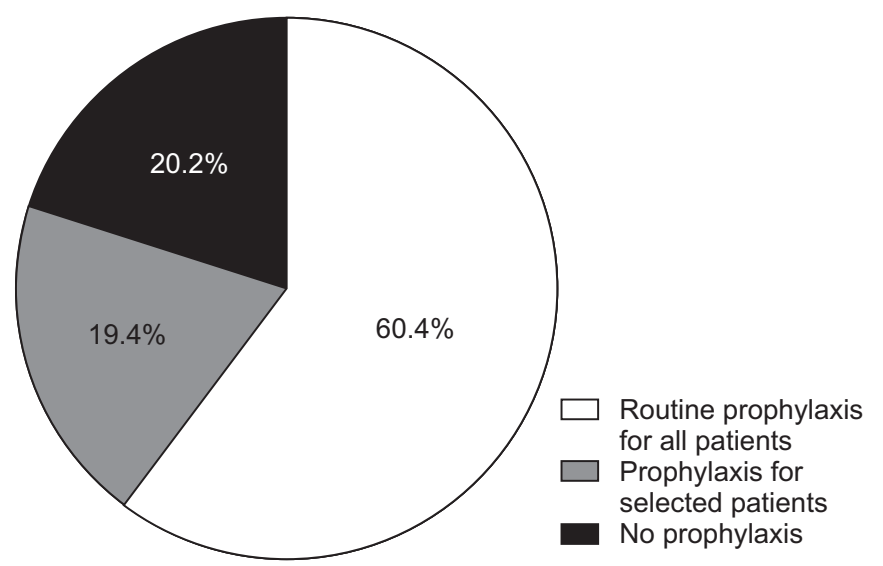

Fig. 2. Pie diagram showing the percentage of orthopedic surgeons who perform prophylaxis for venous thromboembolism after total knee arthroplasty.

therapy and lack of knowledge of the prophylaxis method were the reasons in $35.6 \%$ and $13.3 \%$ of the surgeons, respectively.

The survey regarding the preferred prophylaxis methods was composed of multiple-choice questions. The most commonly used prophylaxis was compression stocking, which was used by $72.9 \%$ of the surgeons who performed prophylaxis, followed by pneumatic leg compression (63.3\%), perioral direct factor Xa inhibitor (46.9\%), and LMWH (39.5\%). In addition, aspirin (20.9\%), foot pump (19.8\%), intravenous factor Xa inhibitor (13.6\%), and warfarin (5.6\%) were applied for VTE prophylaxis after TKA (Fig. 3). Regarding the type of prophylaxis classified as pharmaceutical therapy (aspirin, warfarin, LMWH, factor Xa inhibitor), compression stocking, and intermittent pneumatic pump (foot pump and calf pump), the majority (34.2\%) of respondents utilized all three types. Among the respondents, $14.4 \%$ used a combination of pharmaceutical therapy and compression stocking, $12.2 \%$ used a combination of pharmaceutical therapy and intermittent pneumatic pump, 9.9\% used a combination of compression stocking and intermittent pneumatic pump, $18 \%$ used only pharmaceutical therapy, 6.3\% used only intermittent pneumatic pump, and 5\% used only compression stocking.

\section{Discussion}

The present study is the first survey-based research on the prophylaxis for VTE currently used by orthopedic surgeons in South Korea. The hypothesis of this study was that more than $50 \%$ of medical practitioners who perform TKA in Korea utilize prophylaxis in order to prevent VTE, with mechanical prevention being the most preferred method. The results of this study were consistent with the hypothesis.

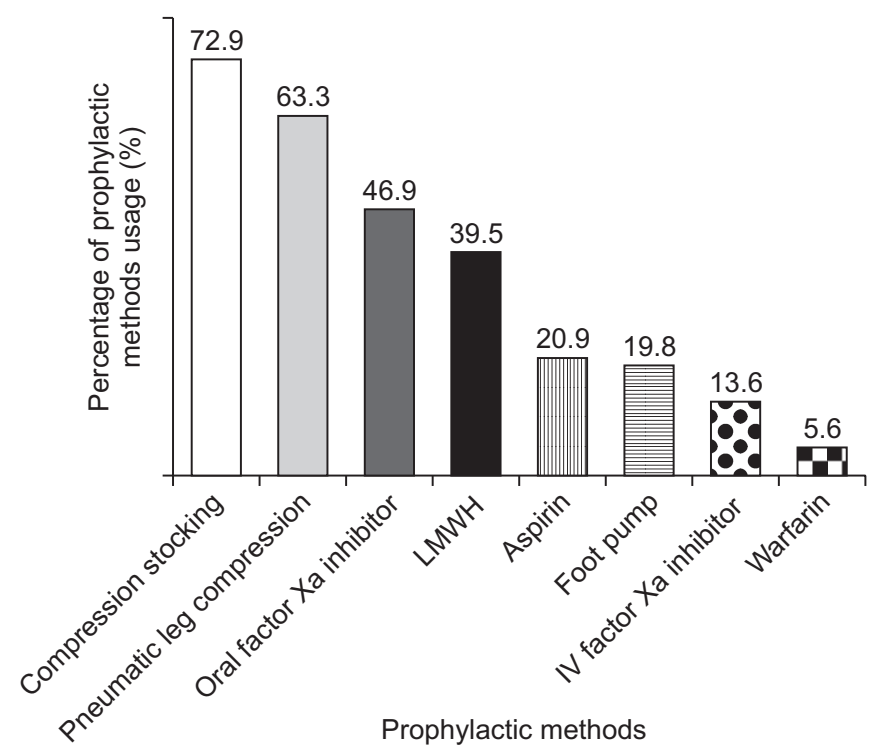

Fig. 3. Bar graph showing the prophylactic methods for venous thromboembolism in the order of usage frequency. LMWH: low-molecularweight heparin, IV: intravenous.

In this survey, the percentage of orthopedic surgeons who routinely performed prophylaxis for venous VTE after TKA was $60.4 \% ; 19.4 \%$ of the surgeons performed prophylaxis depending on the patient's health condition; and the remaining $20.2 \%$ surgeons never implemented prophylaxis after surgery. The main reason for not performing prophylaxis was that the risk of postoperative VTE was judged as low considering the low incidence of VTE. The most commonly used prophylaxis was compression stocking, which was used by $72.9 \%$ of the surgeons who performed prophylaxis, and $34.2 \%$ of the respondents used all the three methods including medical therapy, compression stocking, and intermittent pneumatic pump on the lower limb as prophylaxis.

VTE is a common complication of the lower extremity or spine that occurs after a major surgery. It is related with long-term morbidity and mortality of patients ${ }^{1,6)}$. However, there is still a lack of consensus regarding the implementation of prophylaxis to prevent VTE after joint arthroplasty. Some suggest it is unnecessary to administer anticoagulants to all patients for several reasons including invasiveness and high cost. In addition, it has been suggested that confirmatory examinations, such as computed tomography venography, be performed selectively in patients who display symptoms, and that anticoagulant treatment be performed only after confirming the diagnosis ${ }^{5}$.

Most DVT are asymptomatic, but they can lead to long-term morbidity to the same extent as symptomatic $\mathrm{DVT}^{6}$; therefore, many orthopedic surgeons recognize the importance of pro- 
phylaxis for VTE. In an attempt to minimize the risk of adverse complications, patients are prescribed mechanical devices or pharmacological anticoagulant therapies after TKA in many hospitals. There are many prophylactic regimens including pharmaceutical agents such as aspirin, warfarin, LMWH, and oral factor Xa inhibitors and mechanical devices such as compressive stocking and intermittent pneumatic compression pump and foot pump ${ }^{9,10,12-15)}$.

According to the present study results, $60.4 \%$ of the surgeons routinely performed prophylaxis for VTE after TKA and 19.4\% performed prophylaxis in consideration of clinical situations. Regarding the method of prophylaxis, mechanical device, including compression stocking and intermittent pneumatic compression, were mostly used because they are associated with a lower risk of complications than pharmaceutical agents. Compression stockings and pneumatic compression pumps are advantageous because they do not pose significant risks to patients compared to pharmacologic prophylaxis that requires patient compliance ${ }^{14)}$. Several reports have investigated the incidence of DVT after TKA in South Korea, although they were not based on surveys of as many medical practitioners as was this study. Kim and Kim ${ }^{6}$ examined the incidence of DVT and pulmonary embolism after bilateral TKA and unilateral TKA, where the incidence of DVT after bilateral TKA was $41.8 \%$, and that after unilateral TKA was $41.4 \%$, with no significant differences between groups. Lee et al. ${ }^{5)}$ reported the incidence of DVT after TKA as $17.6 \%$ in patients at high risk for DVT and $14.9 \%$ in low-risk patients.

Several other countries have conducted survey studies on prophylaxis provided by orthopedists to prevent VTE following TKA. Gross et al. ${ }^{11)}$ implemented a survey on the type and frequency of prophylaxis against VTE after TKA and total hip arthroplasty among Canadian orthopedists. In that study, $54 \%$ of the participants responded to the survey compared to $27.9 \%$ in our study. In Canada, $90.2 \%$ of doctors routinely provided prophylaxis for all patients, while only $0.8 \%$ did not provide postoperative prophylaxis. In terms of individual treatments, the largest proportion of respondents (46.3\%) used warfarin, 35.5\% used LMWH, while $40 \%$ used a combination of pharmacological and mechanical methods. Of the mechanical prophylaxis methods, the elastic compression stocking was most widely used, followed by intermittent pneumatic compression. However, only $1.5 \%$ of the doctors used mechanical prophylaxis alone without medication. In comparison to these results of previous studies, the present study showed that the proportion of doctors who routinely provided prophylaxis after TKA in Canada was $90.2 \%$, which is markedly higher than that in Korea (60.4\%). In Canada, the most common method was pharmaceutical prophylaxis using warfa- rin (46.3\%), whereas a low proportion of doctors in South Korea used warfarin (5.6\%). Although a low proportion of doctors preferred mechanical prophylaxis in Canada, the elastic compression stocking was the most commonly used mechanical method, a finding that is consistent with that in Korea.

There are several limitations of this study. The surveyed members of KKS do not constitute the entire practicing orthopedic surgeons who perform TKA in South Korea because only 27.9\% of the surgeons responded to the survey. Therefore, it is possible that the group that participated in this survey may not be entirely representative of surgeons performing joint arthroplasties in Korea and our results may not reflect the actual current status of prophylaxis.

Second, because the present study was based on the memory and experiences of the respondents, the results may not accurately reflect the actual prevalence of the use of prophylaxis therapy.

\section{Conclusions}

The present study is the first survey-based research on the actual status of prophylaxis for VTE in South Korea. The survey showed that $79.8 \%$ of the orthopedic surgeons performed prophylaxis after TKA to prevent DVT and compression stocking was the most common method of prophylaxis.

The present study performed with surgeons who were members of the KKS will help to comprehend the actual status of the prevention of VTE in South Korea and to design VTE guidelines appropriate for Korean patients in the future.

\section{Conflict of Interest}

No potential conflict of interest relevant to this article was reported.

\section{References}

1. Heit JA. Venous thromboembolism: disease burden, outcomes and risk factors. J Thromb Haemost. 2005;3:1611-7.

2. Bjornara BT, Gudmundsen TE, Dahl OE. Frequency and timing of clinical venous thromboembolism after major joint surgery. J Bone Joint Surg Br. 2006;88:386-91.

3. Stulberg BN, Insall JN, Williams GW, Ghelman B. Deep-vein thrombosis following total knee replacement: an analysis of six hundred and thirty-eight arthroplasties. J Bone Joint Surg Am. 1984;66:194-201.

4. Morrey BF, Adams RA, Ilstrup DM, Bryan RS. Complica- 
tions and mortality associated with bilateral or unilateral total knee arthroplasty. J Bone Joint Surg Am. 1987;69:484-8.

5. Lee JS, Kim TW, Suh JT. Deep vein thrombosis after total knee arthroplasty: correlation between the incidence and clinical risk factors. J Korean Knee Soc. 2010;22:270-7.

6. Kim YH, Kim JS. Incidence and natural history of deep-vein thrombosis after total knee arthroplasty: a prospective, randomised study. J Bone Joint Surg Br. 2002;84:566-70.

7. Eikelboom JW, Quinlan DJ, Douketis JD. Extended-duration prophylaxis against venous thromboembolism after total hip or knee replacement: a meta-analysis of the randomised trials. Lancet. 2001;358:9-15.

8. Bergqvist D, Benoni G, Bjorgell O, Fredin H, Hedlundh U, Nicolas S, Nilsson P, Nylander G. Low-molecular-weight heparin (enoxaparin) as prophylaxis against venous thromboembolism after total hip replacement. N Engl J Med. 1996; 335:696-700.

9. Bryson DJ, Uzoigwe CE, Braybrooke J. Thromboprophylaxis in spinal surgery: a survey. J Orthop Surg Res. 2012;7:14.

10. Cohen AT, Tapson VF, Bergmann JF, Goldhaber SZ, Kakkar AK, Deslandes B, Huang W, Zayaruzny M, Emery L, Anderson FA Jr; ENDORSE Investigators. Venous thromboembo- lism risk and prophylaxis in the acute hospital care setting (ENDORSE study): a multinational cross-sectional study. Lancet. 2008;371:387-94.

11. Gross M, Anderson DR, Nagpal S, O'Brien B. Venous thromboembolism prophylaxis after total hip or knee arthroplasty: a survey of Canadian orthopedic surgeons. Can J Surg. 1999;42:457-61.

12. Stewart DW, Freshour JE. Aspirin for the prophylaxis of venous thromboembolic events in orthopedic surgery patients: a comparison of the AAOS and ACCP guidelines with review of the evidence. Ann Pharmacother. 2013;47:63-74.

13. Fuji T, Fujita S, Kawai Y, Nakamura M, Kimura T, Kiuchi Y, Abe K, Tachibana S. Safety and efficacy of edoxaban in patients undergoing hip fracture surgery. Thromb Res. 2014; 133:1016-22.

14. Jaffer AK. An overview of venous thromboembolism: impact, risks, and issues in prophylaxis. Cleve Clin J Med. 2008; 75 Suppl 3:S3-6.

15. Della Valle CJ, Steiger DJ, Di Cesare PE. Thromboembolism after hip and knee arthroplasty: diagnosis and treatment. J Am Acad Orthop Surg. 1998;6:327-36. 


\section{Kim et al. Korean Survey on Prophylaxis for Venous Thromboembolism Following TKA}

Appendix 1. Six-item Questionnaire Mailed to All Members of the Korean Knee Society to Survey the Actual Status of Venous Thromboembolism (VTE) Prophylaxis after Total Knee Arthroplasty (TKA)

In this questionnaire, The VTE means both deep venous thrombosis and pulmonary embolism.

1. What is the form of your working institution and position?

(1) Professor of university hospital in orthopedic surgery

(2) Fellow of university hospital in orthopedic surgery

(3) Specialist of general hospital in orthopedic surgery

(4) Specialist of the specialized hospital in orthopedic surgery

(5) Local orthopedic clinic

2. How many cases of TKA do you operate in a year?

Write down an approximate number, and select an answer from the following list.

$* * *(\quad)$ cases in a year

(1) Less than 20 cases

(2) 20-50 cases

(3) $51-100$ cases

(4) 101-300 cases

(5) More than 301 cases

3. How many cases of TKA have you ever operated until now?

Write down an approximate number, and select an answer from the following list.

***Total ( ) cases

(1) Less than 50 cases

(2) $50-100$ cases

(3) $101-300$ cases

(4) 301-500 cases

(5) More than 501 cases

4. Do you conduct any preventive treatment of VTE after TKA now?

(1) No, I don't conduct any preventive treatment.

(2) Yes, I do, but case by case.

(3) Yes, I always conduct preventive treatment.

5. If you don't conduct any preventive treatment of VTE, what is the reason? (only for those who selected (1) in the question no. 4)

(1) We don't need preventive treatment because the frequency of VTE is low, so the risk of VTE is lower than the risk of preventive treatment of VTE in our country.

(2) I paused preventive treatment because I experienced severe complication of preventive treatment of VTE (bleeding, wound complication, infection).

(3) I want to do, but I don't know detailed method of preventive treatment of VTE.

(4) I have never thought about preventive treatment of VTE.

6. Which preventive treatment of VTE do you conduct? Select every method from following examples.
(1) Aspirin
(2) Warfarin
(3) Low molecular weight heparin
(4) Injection of factor Xa inhibitor (fondaparinux)
(5) Oral direct factor Xa inhibitor (rivaroxaban)
(6) Compression stocking
(7) Foot pump
(8) Intermittent pneumatic compression (calf or calf \& thigh)
(9) Miscellaneous ( )

Thank you for your efforts. 